\title{
AMENDMENTS
}

\section{Author Correction: Programmable RNA editing by recruiting endogenous ADAR using engineered RNAs}

Liang Qu (D), Zongyi Yi, Shiyou Zhu, Chunhui Wang, Zhongzheng Cao, Zhuo Zhou, Pengfei Yuan, Ying Yu, Feng Tian, Zhiheng Liu (1), Ying Bao, Yanxia Zhao and Wensheng Wei 1

Correction to: Nature Biotechnology https://doi.org/10.1038/s41587-019-0178-Z, published online 15 July 2019.

In the version of this article initially published, a relevant work was not cited properly. To correct this error, the first sentence of the second paragraph has been changed from "All these reported nucleic acid editing methods in mammalian systems rely on ectopic expression of two components: an enzyme and a gRN" to "All these reported nucleic acid editing methods in mammalian systems rely almost exclusively on ectopic expression of two components, an enzyme and a gRNA, although Katrekar et al. ${ }^{27}$ have obtained preliminary evidence for editing in the absence of ADAR overexpression." In addition, ref. 42 has been changed from "Abudayyeh, O. O. et al. C2c2 is a single-component programmable RNA-guided RNA-targeting CRISPR effector. Science 353, aaf5573 (2016)" to "East-Seletsky, A. et al. Two distinct RNase activities of CRISPR-C2c2 enable guide-RNA processing and RNA detection. Nature 538, 270-273 (2016)." Finally, $\operatorname{arRNA}_{70}$ has been changed to arRNA 111 in the second-to-last sentence of the sixth paragraph: "The quantitative PCR (qPCR) analysis showed that arRNA 111 did not perturb the expression of targeted transcripts (Supplementary Fig. 3), ruling out the possible RNA interference (RNAi) effect of the arRNA." The errors have been corrected in the HTML and PDF versions of the article.

Published online: 25 September 2019

https://doi.org/10.1038/s41587-019-0292-y

\section{Author Correction: Data privacy in the age of personal genomics}

\section{Dennis Grishin (D, Kamal Obbad and George M. Church (D)}

Correction to: Nature Biotechnology https://doi.org/10.1038/s41587-019-0271-3, published online 19 September 2019.

In the version of this article initially published, an acknowledgement was missing: The authors thank J. Lunshof for critical discussion and feedback on the manuscript. The error has been corrected in the HTML and PDF versions of the article.

Published online: 7 October 2019

https://doi.org/10.1038/s41587-019-0304-y

\section{Author Correction: Precision electronic medicine in the brain}

Shaun R. Patel $\mathbb{D}$ and Charles M. Lieber ${ }^{\mathbb{D}}$

Correction to: Nature Biotechnology https://doi.org/10.1038/s41587-019-0234-8, published online 2 September 2019.

In the version of this article initially published, ref. ${ }^{80}$ was cited in the sentence "Work from our laboratory (C.M.L.) has shown that cells adhere and migrate along the electronically active scaffold of mesh electronics." The correct reference is ref. ${ }^{9}$. The error has been corrected in the HTML and PDF versions of the article.

Published online: 24 October 2019

https://doi.org/10.1038/s41587-019-0319-4 